\title{
Circuit
}

Musiques contemporaines

\section{Bibliographie chronologique des textes et travaux consacrés à Gilles Tremblay}

\section{Marie-Thérèse Lefebvre}

Volume 6, numéro 1, 1995

Tremblay/Varèse/Messiaen : Gilles Tremblay analyste

URI : https://id.erudit.org/iderudit/902122ar

DOI : https://doi.org/10.7202/902122ar

Aller au sommaire du numéro

Éditeur(s)

Les Presses de l'Université de Montréal

ISSN

1183-1693 (imprimé)

1488-9692 (numérique)

Découvrir la revue

Citer ce document

Lefebvre, M.-T. (1995). Bibliographie chronologique des textes et travaux consacrés à Gilles Tremblay. Circuit, 6(1), 57-64.

https://doi.org/10.7202/902122ar d'utilisation que vous pouvez consulter en ligne.

https://apropos.erudit.org/fr/usagers/politique-dutilisation/ 


\section{Gilles Tremblay : documentation}

\section{Bibliographie chronologique des textes et travaux consacrés à Gilles Tremblay Marie-Thérèse Lefebvre}

DE RIGAULT, J. (1954), « Honneur au courage ", Journal musical canadien, vol. $1, n^{\circ} 1$.

GINGRAS, C. (1954), « Les jeunes audacieux de la musique », La Presse, 10 avril.

VALLERAND, J. (1954), \& Garant, Morel ef Tremblay et la musique contemporaine », Le Devoir, 3 mai.

GINGRAS, C. (1954), * Les révélations de l'année musicale à Montréal sont dues à des Canadiens», Culture, vol. XV.

DUVAL, G. (1954), « Trois compositeurs ${ }^{\prime}$ Notre temps, 8 mai.

Anonyme, (1961), « Concert de musique concrète aux JMC \#, Le Devoir, 29 juillet.

MARTIN-DUBOST, P. (1962), "À la tête de prove de la jeune musique canadienne: Gilles Tremblay ", Le Devoir, 4 juillet.

VALLERAND, C. (1962), "Nos "Mozart " assassinés», Le Devoir, 31 juillet.

MARTIN-DUBOST, P. (1963), « Cantique de durées de Gilles Tremblay Vie des arts, $n^{\circ} 31$, p. 45.
MARGROFF, Y. (1967), « Sonorisation du pavillon du Québec", Le Devoir، 16 février.

POTVIN, G. (1967), * Révélation de Tremblay avec Kekoba», La Presse, 6 avril.

MORISSETTE, B. (1967), * L'âme du pavillon du Québec », La Patrie, 11 juin.

THÉRIAULT, J. (1968), * Au premier souffle, c'est la vie: au terme de la mort, l'expiration : création de Champs II. Souffles à la S.M.C.Q.», Le Devoir, 16 mars.

BÉDARD, R. (1968), \& L'Homme est semblable à un souffle... *, Sept-Jours, 24 mars.

Anonyme, (1 968), * Gilles Tremblay remporte le prix Calixa-Lavallée », Le Devoir, 27 avril.

TREMBLAY, D. (1968), « Gilles Tremblay, ex-élève de Maurice Martenot », Montréal Matin, 9 mai.

POTVIN, G. (1968), * La carrière musicale d'un lauréat, Gilles Tremblay », La Presse, 11 mai.
MAITRE, M. (1968), « Le compositeur Gilles Tremblay déçu de voir sa sonorisation supprimée au pavillon du Québec $»$, La Patrie, 30 juin.

THÉRIAULT, J. (1970), «Gilles Tremblay », La Scène musicale, $n^{\circ} 252$, pp. 6-7.

THÉRIAULT, J. (1970), \& Du vent, des premiers pas, un film et des tambours", Le Devoir, 24 octobre.

GARANT, S. animateur (1971), présentation de Kekoba, de Gilles Tremblay, émission Musique de notre siècle, RadioCanada, réalisateur Claude Garneau.

KENDERGI, M. (1972), Cahier du musialogue Gilles Tremblay, Université de Montréal.

THÉRIAULT, J. (1972), * Gilles Tremblay aux musialogues: à l'écoute de l'Orient, paradis de la musique», Le Devoir, 16 décembre.

GARANT, S. animateur (1972), présentation de Souffles-Champs II de Gilles Tremblay, émission Musique de notre siècle, Radio-Canada, réalisateur Claude Garneau. 
GAGNON, M. ef LAFONTAINE, L. (1973), Dossier Gilles Tremblay, École normale de musique.

GARANT, S. animateur (1973), présentation de Champs / et Le Sifflement des vents porteurs de l'amour... de Gilles Tremblay, émission Musique de notre siècle, Radio-Canada, réalisateur Claude Garneau.

THÉRIAULT, J. (1974), « Journées de musique à voir », Le Devoir, 8 juin.

THÉRIAULT, J. (1974), " Les JMC fêtent leurs 25 ans à Orford", Le Devoir, 24 juillet.

THÉRIAUIT, J. (1974), « Un outil précieux, les "Compositeurs au Québec" ", Le Devoir, 24 septembre.

LAPLANTE, L. dir. (1974) (révisions 1982 et 1991), Gilles Tremblay, Série "Compositeurs au Québec ", Montréal, Centre de musique canadienne.

RICHARD, R. (1975) (révision 1980 et 1988), Gilles Tremblay, Montréal, SDE.

ROBERGE, J. (1975), Gilles Tremblay, sa vie, son œuvre, Mémoire présenté au Concours d'histoire de la musique et musicologie, Conservatoire de Musique du Québec.

BILLETTE, G. (1976): Analyse de Kekoba, série « À l'écoute de la musique d'ici $», n^{\circ} 1$, Montréal, Centre de musique canadienne.

POTVIN, G. (1976), "Fleuves en création mondiale ", Le Devoir, $1^{\text {er mai. }}$

GINGRAS, C. (1976), \&'OSM retire l'œuvre de Tremblay de son programme », La Presse, 7 mai.
POTVIN, G. (1976), De Burgos annule Fleuves commandé à Gilles Tremblay », Le Devoir, 7 mai.

Anonyme, (1976), "MSO Cancels of Postpones Tremblay's Work», The Gazette, 7 mai.

Anonyme, (1976), * MSO Changes Plans », The Montreal Star Entertainments, 8 mai.

McLEAN, E. (1976), * Music Council Meet Focuses on Economy», The Montreal Star, 13 mai.

RYAN, C. (1976), "Une victime douce mais combien éloquente ", Le Devoir, 21 mai.

POTVIN, G. (1976), « Une superbe fête musicale marque le $10^{\mathrm{e}}$ anniversaire de la SMCQ: création de Oralleluiants», Le Devoir, 13 décembre.

Anonyme, (1977), " Montreal Symphony Lines up Conductors», The Montreal Star, 4 janvier.

SAMSON, M. (1977), "Musique », le Soleil, 13 janvier.

SISKIND, J. (1977), «MSO Readies Contemporary Scores», The Gazette, 27 avril.

GINGRAS, C. (1977), «Fleuves de Tremblay: comme au ballon-panier $»$, entrevue avec Garant et Tremblay, La Presse, 30 avril.

POTVIN, G. (1977), « Fleuves de Gilles Tremblay, enfin créé !», Le Devoir, 30 avril.

MCLEAN, E. (1977), \& Flood of Discord Greets New Work», The Montreal Star, 4 mai.
SISKIND, J. (1977), *World Premiere of Fleuves Makes Light of all its Hazards », The Gazette, 4 mai.

POTVIN, G. (1977), « Une soirée stimulante ", Le Devoir, 5 mai.

SISKIND, J. (1977), «Fleuves Must be More than a Costly Modern Music Lesson », The Gazette, 7 mai.

McLEAN, E. (1977), Fleuves in Retrospect », The Montreal Star, 7 mai.

GINGRAS, C. (1977), *Tremblay et Garant: un double événement *, La Presse, 8 mai.

PRÉVOST, P. (1977), « Fleuves de Gilles Tremblay, une date mémorable pour I'OSM », le Jour, 13 mai.

RICHARD, R. (1975), |révisions 1980 et 1988), "Gilles Tremblay », brochure SDE.

RICHARD, R. (1977)، « Gilles Tremblay » Vie des Arts, n० 86, pp. 58-59.

MATHER, B. (1 977), « Gilles Tremblay » in L. LAPLANTE, (dir.), Compositeurs canadiens contemporains, Montréal, Presses de l'Université du Québec, pp. 337. 341 .

BAll-MILOT, L., animatrice (1979): La Musique de Gilles Tremblay, émission Musique de Canadiens, Radio-Canada, réalisateur: Claude Ouellet, 22 juin.

BAIL-MILOT, L., animatrice (1980) : Une analyse de Kekoba de Gilles Tremblay, émission Musique de Canadiens, RadioCanada, réalisateur: Jean Morin, 2 octobre.

MATHER, B. (1980), * Gilles Tremblay * in SADIE, St. ed., Grove's Encyclopedy 
of Music and Musicians, vol. 19, Londres, McMillan Publ.

RICHARD, R. (198 1), " Gilles Tremblay *, in $\mathrm{H}$. RALLMANN, G. POTVIN, et $\mathrm{H}$. WINTERS, Encyclopedy of Music in Canada, Toronto, Toronto University Press.

POTVIN, G. (1981), "DZEI, une autre œuvre magistrale de Tremblay», Le Devoir, 30 septembre.

BAIL-MILOT, L., animatrice (1982), Gilles Tremblay: vivre l'Orient, émission Musique de Canadiens, Radio-Canada, réalisateur: Armand Plante, 16 mai.

En collaboration, (1982), «Propos sur Gilles Tremblay à l'occasion de son $50^{e}$ anniversaire », Sonances, vol. $2, n^{\circ} 1$, pp. 19-28.

HELFFER, C. (1983) : Notes sur Traçantes, auprès, au loin... 1976, programme de la SMCQ, 17 février.

POTVIN, G. (1983), «Une semaine Gilles Tremblay », Le Devoir, 12 février.

GINGRAS, C. (1983), *Création d'Envoi de Gilles Tremblay», La Presse, 17 février.

BERGERON, C. (1983), "Tremblay: musique de l'émerveillement », Le Devoir, 19 février.

URQUHART, C. (1983), «A Fine Tribute to Tremblay», The Gazette, 19 février.

PANNETON, I. (1983), « Semaine Gilles Tremblay à Montréal », Sonances, vol. 2, n० 3, pp. 23-24.

VILLENEUVE, A. (1983), "Souffles (Champs II) de Gilles Tremblay », travail pour le concours d'analyse, Conservatoire de musique de Montréal.
Anonyme, (1986), « Création québécoise à l'Abbaye de Sylvanes: Les Vêpres de Gilles Tremblay», La Presse, 14 juillet.

LONGCHAMPT, J. (1986), * la tradition revigorée: Les Vêpres de Gilles Tremblay à l'Abbaye de Sylvanes », Le Devoir, 24 juillet.

GINGRAS, C. (1987), * Les Vêpres de Gilles Tremblay: du grégorien au son du gong », La Presse, 18 juillet.

TÉTREAU, P. (1987), «Vêpres accomplies », Spirale, septembre.

BERGERON, C. (1987), « Sublime, l'œuvre de Gilles Tremblay évoque Monteverdi : Les Vêpres de la Vierge au festival de Lanaudière », Le Devoir, 18 juillet.

BERGERON, C. (1987), «Ovation pour un deuxième miracle: Les Vêpres de la Vierge à Lanaudière », Le Devoir, 21 juillet.

McLEAN, E. (1987), «Tremblay Premiere Draws Large Crowd „, The Gazette, 21 juillet.

GINGRAS, C. (1987), * Une grande reprise: Les Vêpres de Tremblay», La Presse, 4 décembre.

BERGERON, C. (1987), * Le fabuleux monde sonore de Gilles Tremblay», Le Devoir, 4 décembre.

SAMSON, M. (1988), " Les Vêpres de la Vierge de Gilles Tremblay : une œuvre sobre, pure et fascinante », Le Soleil, 27 février.

BOIVIN, J. réalisateur, (1988) : Les Cours d'été à Darmstadt, émission Musiques actuelles, Radio-Canada, 3 juillet.

LITTLER, W. (1988), \& BBC Scottish Scores With Gilles Tremblay: Katadrone
(Contrecri) », The Toronto Star, 24 octobre.

OUELLETTE, F. (1988), L'Oreille intérieure (hommage à Gilles Tremblay), in F. Ouellette, Ouverture, Montréal, L'Hexagone/Librairie Bleve, pp. 146-147.

GAGNÉ, M., LAUBER, A., MARTIN, G., RIOUX, A., ROBITAILLE, G. (1990) : Sons d'aujourd'hui, Montréal, Louise Courteau éditrice, livre de l'élève ; pp. 135-139, livre du maître; pp. 125-132, cassette $n^{\circ} 19$.

LAVOIE, P. Y., réalisateur, (1990): De musique en musiques; Gilles Tremblay, Accès vision 9 et Câble TV Inc.

LAURIER, M. (1990), "Gilles Tremblay: en toute cohérence et avec harmonie ", Le Devoir, 12 octobre.

MARCOTTE, G. (1992), Jubilation (notes sur Gilles Tremblay), in L'Amateur de musique, Montréal, Boréal, pp. 87-93.

LAURIER, M. (1992), « Une œuvre de Gilles Tremblay en première mondiale à Radio-Canada ", Le Devoir, 10 avril.

DUBOIS, A., réalisateur (1992), * Hommage à Gilles Tremblay pour son $60^{\mathrm{e}}$ anniversaire " émission Musique en fête, Radio-Canada, 7 septembre.

GINGRAS, C. (1992), *Gilles Tremblay: une création radiodiffusée en direct dans 13 pays européens», la Presse, 2 octobre.

BRETON, B. (non daté), Analyse de Phases (1956) de Gilles Tremblay, fonds d'archives Serge Garant P. 141, C4.7, Service des archives de l'Université de Montréal. 
GARANT, S. (non daté), Notes pour l'analyse de Souffles (Champs III incluant des notes de Gilles Tremblay, fonds d'archives Serge Garant, P 141, C3.13, Service des archives de l'Université de Montréal.

GARANT, S. (non datél, Notes pour l'analyse de Vers (Champs III) incluant des notes de Gilles Tremblay, fonds d'archives Serge Garant, P 141, C3.14, Service des archives de l'Université de Montréal
LAURIER, M. (1992), "Avec de Gilles Tremblay à Radio-Canada», Le Devoir, 9 octobre.

GINGRAS, C. (1992), « Avec, création d'une œuvre de Gilles Tremblay sur la fondation de Montréal », La Presse, 10 octobre.

GINGRAS, C. (1992), * Gilles Tremblay et son wampum », La Presse, 13 octobre. BERGERON, C. (1992), "Une œuvre monumentale», Le Devoir, 14 octobre.
BERGERON, C. (1992), * Une mémorable soirée d'anniversaire " Le Devoir, 29 octobre.

VILLENEUVE, A. (1992), * Notation ef usage du mobile chez Gilles Tremblay ", travail pour le « Séminaire de doctoral », Faculté de musique, université McGill.

Anonyme, (1993), * Artiste pour la Paix 1992 » La Presse, 14 février. 
61

Vous tonverez sous ple les tin's étapes de latiauseriftion f'une éconce de bon bau.

I. Contumplation de l'écra

II. Décalque sus papier nuillinuitror (ici, égérenenent práuit).

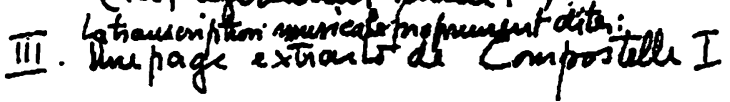

pour 18 exécutants. L'éconce commence an chiffe 14 Elle est confies à 2 flüts et 3 violons en quast de ton's, ainsi qu'à's galets et 4 wood - blocks.

Biensuis on promait l'intrepreites de bien d'autss facoses. A qui mia frap/w c'est l'aspect imusical de l'éence sailér en partitini, avee ses lignes haizon tales de différeuts longeneurs et ses gionpes-réseaux. d'éveruements, comme au constellatiois vihatiles. (Le champrs des etriles.).

voilà pour votre herbier, dela part

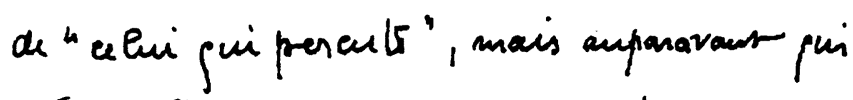
s'émerveille. Là in science et ant se sejoiquect en poésic, en contemplatioñ.

Trsamicalmuent,

Gilles. T. 


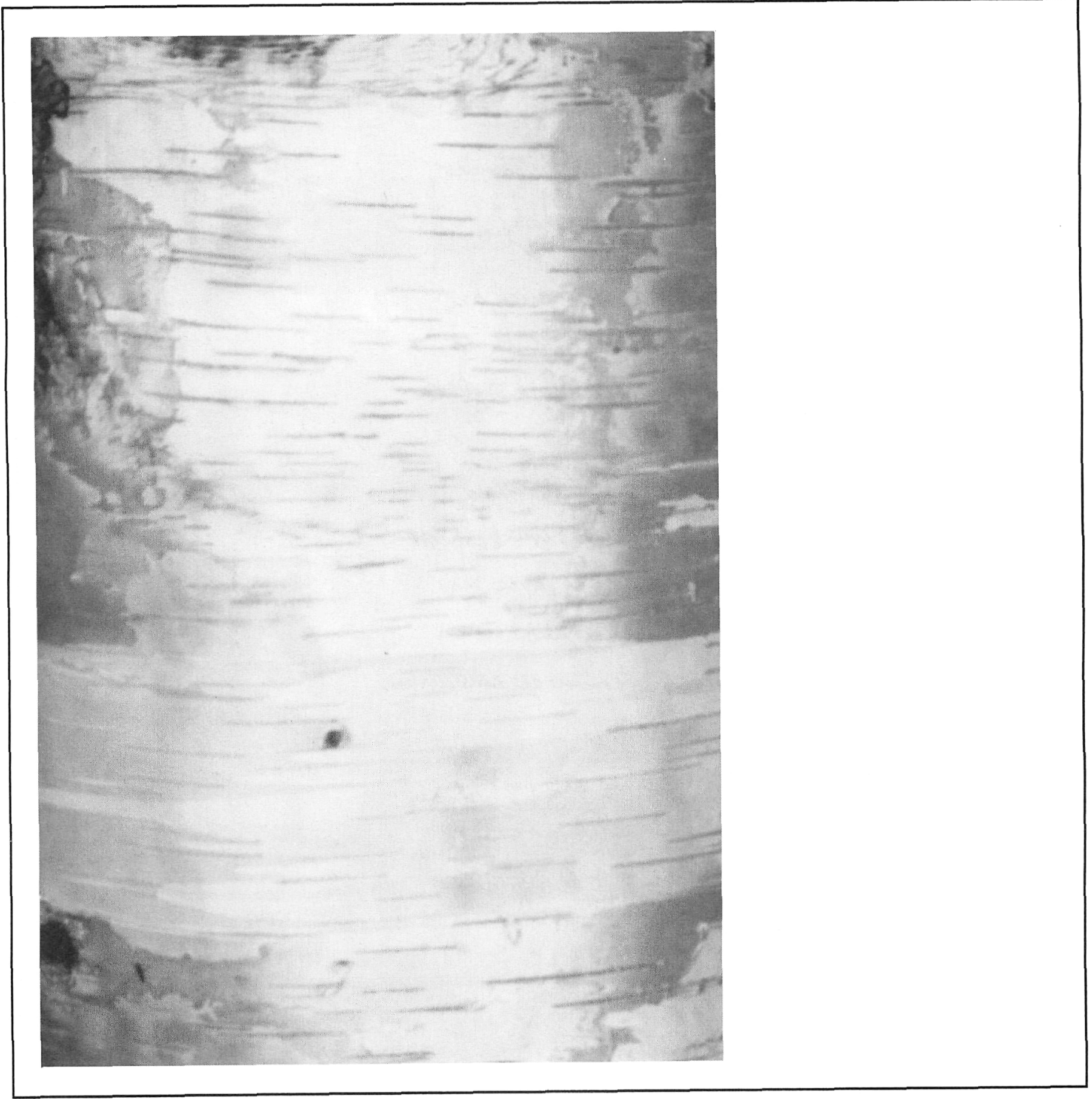




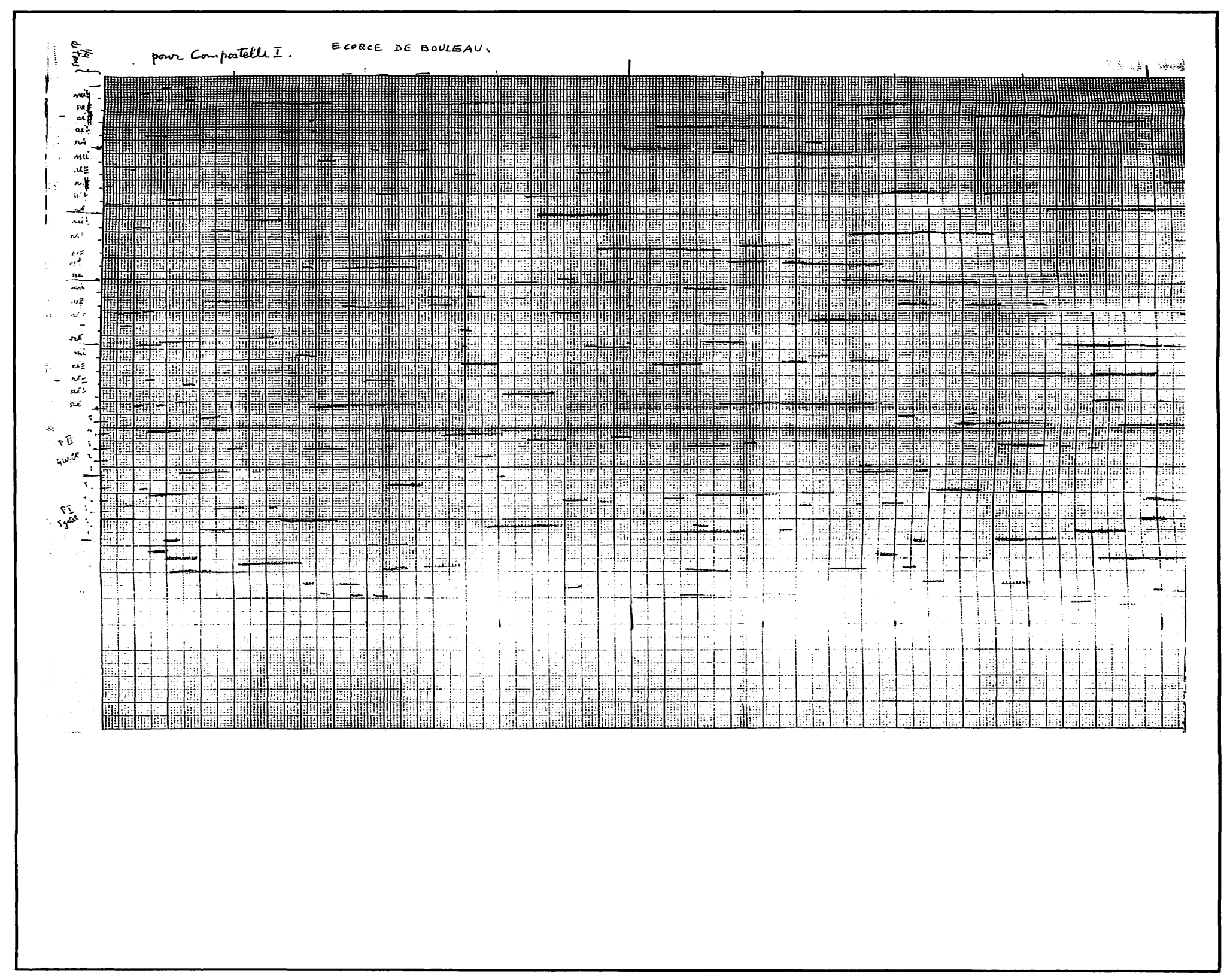




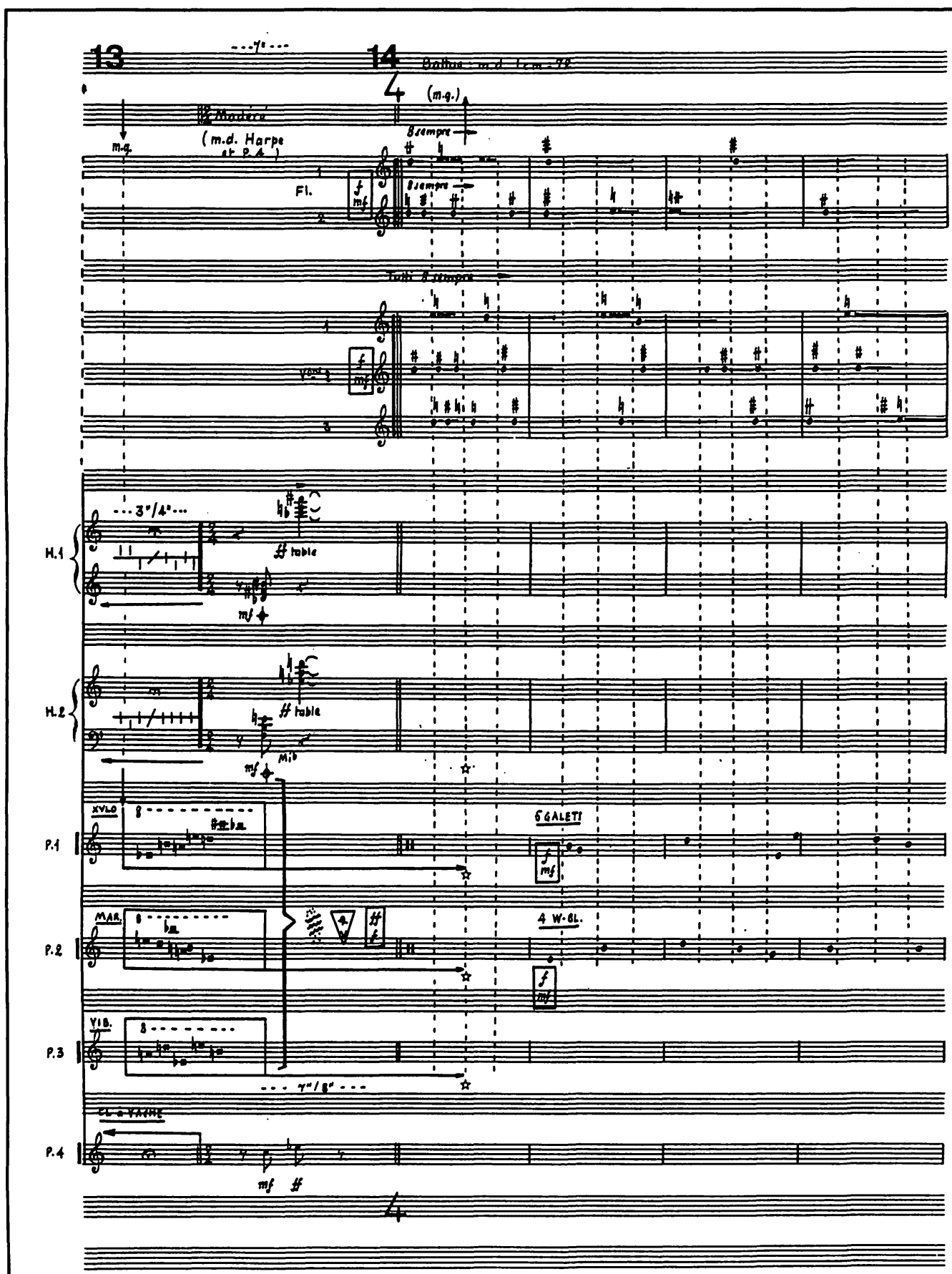

